\title{
Improving Take-Over Quality in Automated Driving By Interrupting Non-Driving Tasks
}

\author{
Thomas Köhn \\ Matthias Gottlieb \\ Chair for Information Systems \\ Technical University of Munich \\ Munich, Germany \\ \{thomas.koehn|gottlieb\}@in.tum.de
}

\author{
Michael Schermann \\ Santa Clara University \\ Santa Clara, CA, United States \\ mschermann@scu.edu
}

\author{
Helmut Krcmar \\ Chair for Information Systems \\ Technical University of Munich \\ Munich, Germany \\ krcmar@in.tum.de
}

\begin{abstract}
With automated driving advancing, first production models started to incorporate the technology. However, until full autonomy is achieved, drivers always need to stay available to take over control from the car. This requirement has proven challenging: increased levels of automation reduce drivers' situational awareness and driving performance can suffer, especially in the critical moments after take-over. While manualdriving research introduced strategies to direct drivers' attention back to the road, notably interruptions of the non-driving task, the efficacy of these interventions on automated driving remain unclear. To investigate this, 53 participants drove in an automated simulator while performing tasks on an IVIS. With task interruptions, they reported increased situational awareness and showed improved reaction times during take-over, particularly for low-effort tasks (watching movies). Different to manual driving, halting tasks did not suffice; instead, we displayed the driving scene. Results question effects of situational awareness on take-over and offer solutions for manufacturers.
\end{abstract}

\section{CCS CONCEPTS}

- Human-centered computing Empirical studies in ubiquitous and mobile computing • Human-centered computing Empirical studies in interaction design • Humancentered computing Information visualization

\section{KEYWORDS}

Semi-automated driving; take over request; situational awareness; out of loop; IVIS; interruptions.

\section{ACM Reference format:}

Thomas Köhn, Matthias Gottlieb, Michael Schermann and Helmut Krcmar. 2019. Improving Take-Over Quality in Automated Driving By

Permission to make digital or hard copies of part or all of this work for personal or classroom use is granted without fee provided that copies are not made or distributed for profit or commercial advantage and that copies bear this notice and the full citation on the first page. Copyrights for third-party components of this work must be honored. For all other uses, contact the Owner/Author.

IUI '19, March 17-20, 2019, Marina del Ray, CA, USA

(C) 2019 Copyright is held by the owner/author(s).

ACM ISBN 978-1-4503-6272-6/19/03.

https://doi.org/10.1145/3301275.3302323
Interrupting Non-Driving Tasks. In 24th International Conference on Intelligent User Interfaces (IUI'19), March 17-20, 2019, Marina del Rey, CA, USA. ACM, New York, NY, USA, 8 pages.

https://doi.org/10.1145/3301275.3302323

\section{Introduction}

More than ten years after the first iterations of the DARPA Grand Challenge were completed [7], full, level-5 driving autonomy [17] remains elusive for the general public. Over the last few years, several manufacturers delivered level-2 models, which recently also became available in the more affordable sub$\$ 40,000$ segments [12]. The first level-3 models are about to be released. These two levels are considered the most critical ones in driving automation, as the car can already take over all operational aspects, like steering, braking and accelerating, but the driver still needs to be able to take over control at any time.

The car triggers a take-over request (TOR) to transfer control to the driver in situations that exceed the limits of the automation (e.g., in [6,14]). For instance, this could be due to a required tactical decision, like diverging roads, or due to unsupported conditions, like missing lane markings or adverse weather [6,35]. The time until the automation suspends varies based on the situation but is usually considered between 4 and 8 seconds [5]. The TOR signal frequently comes in visual and auditory form [37], but may also be haptic [30]. To take over control, drivers usually only need to interact with the steering wheel or foot pedals.

Several studies indicate, however, that especially in the critical moments after take-over, the driver's performance is diminished $[23,33]$. Issues range from operational shortcomings, like delayed reactions for braking or steering, high variability in driving speed, and increased steering angles, to tactical errors, such as changes to the wrong lane. Drivers' situational awareness seems affected as well [23]. This issue constitutes common problems of automation, not restricted to the automotive domain, in which operators are out of practice due to the lack of motor control; and their information consumption switches from active to passive [11]. Additional, new distractions, e.g., information systems, further amplify the effect.

In this work, we aim to improve situational awareness and driving performance immediately after take-over by modulating 
a common distractor in the car. Assuming that people will spend much time with non-driving related information consumption and similar tasks, we focus our efforts on in-vehicle information systems (IVIS). As manual driving research has already proposed several suggestions on how to direct the driver back to the road, we will investigate the applicability of one of them to automated driving, namely interruptions of IVIS usage [8]. Based on this, we introduce two different treatments: interruptions of the IVIS with a blank screen, and interruptions with the driving scene. We investigate the effect of these treatments on two types of tasks: passive, low-effort tasks, and active, cognitively demanding tasks, as previous research points to different effects (e.g., [19]). With this setup, we performed a high-fidelity driving simulator experiment with 53 participants.

We hypothesize that both types of interruptions will have a positive effect on driving performance and situational awareness, with a more pronounced effect for interruptions with the driving scene. We also expect some effect from blank-screen interruptions, as they may prompt subjects to look up to the road again. Task performance should not be affected, as we keep interruptions short.

- H1 Reaction Time: Interruptions will significantly decrease the driver's reaction time to take-over requests, especially when interruptions are augmented with the current driving scene.

- $\quad$ H2 Situational Awareness: Interruptions will significantly increase the driver's situational awareness of the driving situation, especially when interruptions are augmented with the current driving scene.

- H3 Task Performance: Interruptions will not significantly influence the driver's task performance and sentiment about task execution.

With this research, we contribute to theory and practice. We extend the body of literature regarding the connection of situational awareness and driving performance after take-over. For manufacturers, our study offers a practical solution that increases road safety.

The remainder of this paper structures as follows: we first review the relevant scientific literature for our study. Then we introduce our study design, including a detailed description of the simulator, TOR scenario, and treatments. Afterward, we present the results from this study and discuss their implications, as well as the study's limitations. We conclude with our suggestions for future research on the topic.

\section{Related Work}

This section structures the background of our study in three parts. We first explain the automation problem, which is found in many domains beyond automotive. Then, we outline existing strategies to improve take-over quality. We conclude with a review of interruption research, both based on the psychological literature and their effects during driving.

\subsection{The Automation Problem}

With an increasing level of automation, the manual performance on the task decreases. Issues include a reduced ability to detect problems, reduced situational awareness, and reduced ability for manual operation in case of system failure [4,11]. The issue is not domain-specific: aviation research indicated already that pilots were not able to safely take over again from automation [4]; pilots thus tended to deliberately switch to manual mode from time to time [39].

Many studies have confirmed the issue as well for driving automation (e.g., [23,33]). Research has looked at the effects of different lead times [14]; how different non-driving tasks affect take-over [32]; and how the driving situation influences the driver [32]. Merat \& Jamson [23] suggest that drivers trust the automation too much.

\subsection{Improving Take-Over Quality}

Previous research has looked at different ways to improve driving performance and situational awareness for take-overs, with strategies ranging from preventive driving measures, to TOR signals, non-driving task interventions. Frequent take-overs can improve quality $[14,21,35]$. Multimodal TOR signals proved beneficial compared unimodal signals [30,31]. Information presentation closer to, or merged with the driving scene showed positive effects [18,20]; and using an IVIS for this is preferred as compared to handheld devices [41]. Closest to our study, Wulf et al. [40] permanently displayed the driving scene in one corner of the IVIS and could improve situational awareness, but not driving performance; Naujoks et al. [27] recommended, but did not study on interruption strategies to keep the driver in the loop.

\subsection{Interruptions}

The effects of task interruption are a well-studied phenomenon in the psychological literature, with both, potential benefits and shortcomings. Interrupting a task may prolong its duration (beyond the time of interruption), and increase task errors [3,38]. The duration and cognitive demand of the interruption play into this, as well as the point in time of the task [3,24-26]. And while a task is unfinished, it may consume additional cognitive resources [42]. However, interruptions can also increase task performance and efficiency at the same level of quality [43].

In manual driving research, interruptions likewise play an essential role in three distinct ways. First, the adverse effect of interruptions of the driving task by non-driving related, unsolicited events, especially from information and communication technology, spurred a whole study area into workload managers [15]. Similarly, second, the interrupted attention to the driving scene stemming from drivers voluntarily engaging in non-driving related tasks has been a focus in research, primarily pointing to the negative effects of different types of tasks (e.g., [19]); they can, however, also have a positive effect on driving performance, if they, e.g., prevent underload $[9,13,28,29]$. Third, and most important to our research, the effects of interrupting the non-driving related task have been 
Improving Take-Over Quality in Automated Driving By Interrupting Non-Driving Tasks

studied, both with soft warnings and hard blockings of the user interface, and results indicated improvements in driving performance and behavioral changes in regard to the source of distraction $[8,22]$.

\section{Method}

To test our hypotheses, we conducted a driving simulator experiment with 53 participants. Each of them performed two interruption-based treatments and a baseline while driving in automated mode on a highway. We measured driving performance during take-over, situational awareness, task performance, load, and user sentiment to compare the conditions.

\subsection{Conditions}

Participants performed a run for each of the three conditions in a counterbalanced design. During the baseline run, they engaged with the non-driving related task without any interventions. In the Scene condition, the non-driving task was interrupted every $30 \mathrm{~s}$ with a live view of the forward driving scene and the mirror views, which lasted for $3 \mathrm{~s}$. In the Blank condition, the nondriving task was interrupted in the same intervals as in the Scene condition, but with the IVIS screen empty.

\subsection{Driving Task \& Track}

The driving task took place on a straight, two-lane highway. The car drove with $120 \mathrm{~km} / \mathrm{h}(\sim 75 \mathrm{mph})$ in automated mode. Traffic conditions were light to medium. Cars are only visible in the same direction, trees and bushes conceal the opposite lanes. Light and weather conditions were normal: it was daytime, and we did not apply any particular weather. The TOR came after about $4 \mathrm{~min}$ of the drive, to bring participants out-of-loop.

For the take-over, the current lane was obstructed with a construction site, which only became visible after a lead vehicle switched lanes. Participants had to switch lanes as well and could modulate the speed. The take-over request was emitted $7 \mathrm{~s}$ before a collision with the construction site, and in both, visual and auditory form by the IVIS [31].

We put several measures in place to prevent learning effects in the course of the three conditions. We used several highway courses that differed in exact traffic but used identical take-over scenarios for comparability. To prevent participants from detecting the take-over scenario prematurely, we, on the one hand, obstructed it as described with a lead vehicle, and on the other hand, we used several identical scenarios throughout the automated drive, which did not trigger a TOR; the number of these non-TOR obstacles also varied.

\subsection{Non-Driving Task}

We used two non-driving related (NDR) tasks for our study: a low-effort, passive task, and a cognitively demanding, active task, both displayed on an IVIS. For the passive task, we used movie watching (referred to as Movie), for which we played
IUI'19, March, 2019, Marina del Ray, California USA

short cartoons without language (Tom \& Jerry). The cartoons lasted about the duration of one drive; we used different ones for different drives and counterbalanced them to prevent potential effects from one specific cartoon. For the active task, we chose a mental rotation task called Little Man Task (LMT) [2]. In the LMT, a figure is displayed with a suitcase in either the left or right hand, and the figure is rotated around different axes. Subjects have to decide in which hand the suitcase is. Subjects either performed the LMT or Movie task (between-subject design).

\subsection{Participants}

Across both tasks, 53 people (12 female) participated in the study, with ages ranging from 19 to $33(M=22.5, S D=2.96)$. We recruited participants from personal contacts, with flyers in the university building, and through announcements in lectures and student-focused online groups. Most participants had their driving license for several years, two had it for less than one year, three had it for 10-14 years $(M=4.5$ years, $M d n=4, S D=$ $2.82)$; estimated mileage per year ranged from a few kilometers up to $30,000 \mathrm{~km}(M=6149 \mathrm{~km}, \sim 3821$ miles; $M d n=2500 \mathrm{~km} ; S D=$ 7908). As compensation, they received snacks. Due to the between-subject design, 27 participants performed the LMT, and 26 the Movie task.

\subsection{Apparatus}

We used a high fidelity driving simulator with OpenDS 4.5 [1] (see Figure 1). The cockpit consists of a Mercedes dashboard. The car has an automatic transmission to ensure that there are no biases due to changing gear [36]. We used the original Mercedes steering wheel with the ClubSport Wheel Base V2 Servo and the ClubSport Pedals V3 from fanatec. The side and back mirrors were displayed in software. The projection system to display the driving scene consists of three projectors with a resolution of each $1280 \times 1024$ pixels. The images are edge-blended to provide a total horizontal view of $120^{\circ}$ with a $6 \mathrm{~m}$ radius on a cylindrical

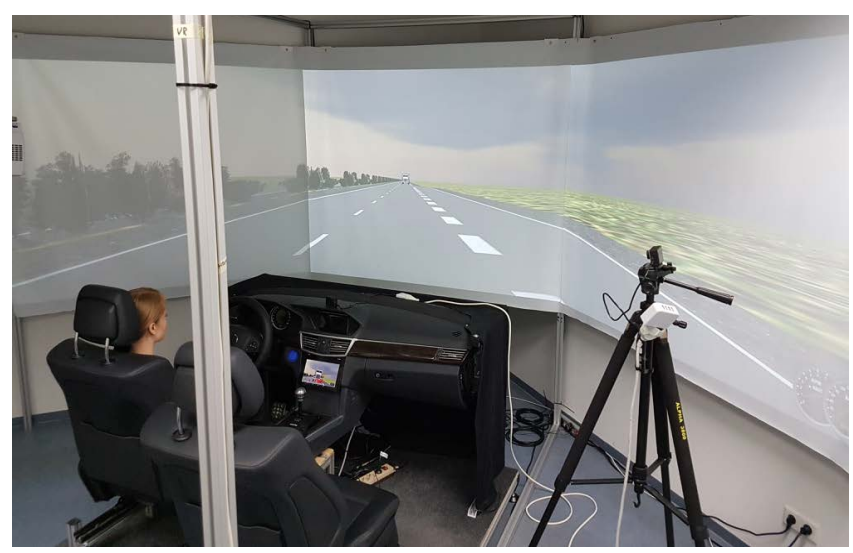

Figure 1: Side view of the driving simulator with its $120^{\circ}$ view. The simulation shows the 2-lane highway, here with a temporary third lane for oncoming traffic from a 
screen with $45^{\circ}$ to each other in front of the driver. The IVIS was a 9-inch tablet, which was mounted in common height in the center console in landscape format.

\subsection{Measures}

For driving performance, we measured participants' reaction time from the moment the TOR signal was triggered until they turned the steering wheel or pressed one of the foot pedals. For task performance, we compared correct and wrong answers in the LMT and used a post-study questionnaire with 7 questions regarding events from the movie. We gauged situational awareness with the SART questionnaire [34]. Finally, we inquired about participants' sentiment towards and load from the non-driving task and interruptions with the NASA-TLX [16].

\subsection{Procedure}

Upon arrival, the experimenter greeted participants, and they filled out a demographic survey. The experimenter then explained the purpose of the study in more detail and introduced participants to the driving simulator. Participants took two sets of familiarization drives: one in a city track to get accustomed to the controls of the simulator, one in a highway situation similar to the study, to get used to the automated mode and to practice take-overs. We performed both until the participant felt comfortable with the controls, which usually took 5-10min. Afterward, the recorded part of the study began, with the identical procedure for each condition: participants drove with either one of the treatments, or in the baseline, and performed the take-over after about $4 \mathrm{~min}$ of driving; after each drive, they filled out the NASA-TLX, SART, and movie questionnaire (the latter unless they were in the LMT group). After completing all three conditions, the experimenter briefly interviewed them about the experiment to get unstructured feedback and debriefed participants. The whole study took about $40 \mathrm{~min}$.

\section{Results}

As the two NDR tasks are very different, we look at them separately for each measure.

\subsection{Driving Performance - Reaction Time}

4.1.1 Movie Task. For the Movie task, we observed significant differences in reaction time (see Figure 2). Participants reacted faster with both, Scene $(M=2.42, S D=1.04)$ and Blank $(M=2.55$, $S D=.83)$ interruptions, as compared to the baseline $(M=2.72$, $S D=.95)$. The sample for baseline and Blank were normally distributed, while the sample for Scene was not. While a Friedman test of differences failed significance $\left(\chi^{2}=4.88\right.$, $p=.08716)$, pairwise Wilcoxon one-tailed tests showed a significant difference between baseline and Scene $(W=226, p=$ $.04516)$ and Scene and Blank $(W=247, p=.01095)$, but not for baseline and Blank ( $W=189, p=.2454)$. We considered but ultimately did not remove two outliers, whose reaction time was slightly below and slightly above $M+3$ * $S D$ : it would have only

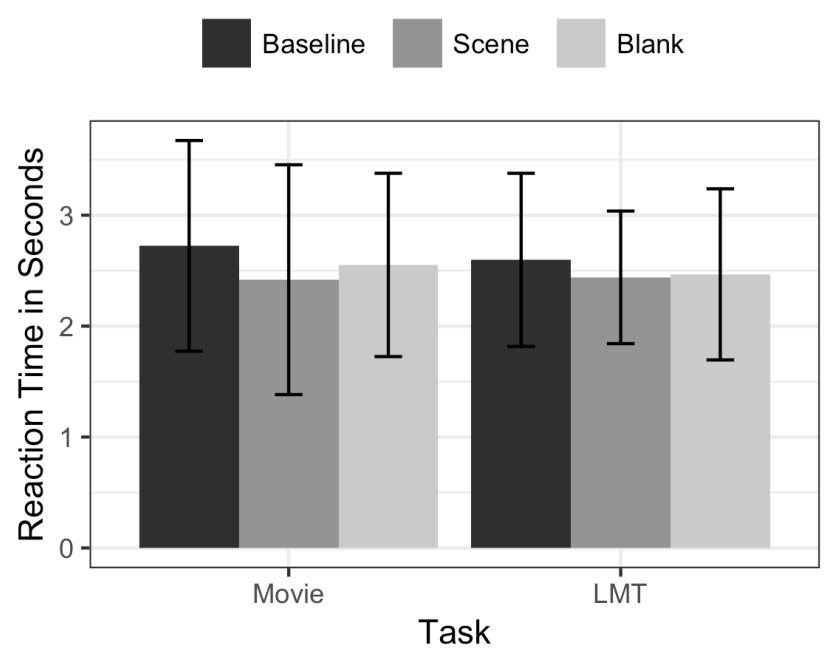

Figure 2. Reaction time after TOR signal for each condition, separated by task; error bars indicate the standard deviation.

affected the overall Friedman, bringing it to significance, while all pairwise tendencies remained the same.

4.1.2 LMT Task. For the LMT task, the reaction time did not show any significant differences (see Figure 2). While again, participants reacted faster with Scene $(M=2.44, S D=.6)$ and Blank $(M=2.47, S D=.77)$ interruptions as compared to the baseline $(M=2.6, S D=.78)$, neither a Friedman test of differences $\left(\chi^{2}=1.1852, p=.5529\right)$, nor pairwise Wilcoxon one-tailed tests for Scene and baseline ( $W=239, p=.1193)$, Blank and baseline $(W=210, p=.3138)$, or Scene and Blank $(W=197, p=.4297)$ showed significance. In this task, outliers were much less pronounced than for the Movie task, and showed no reason for removal.

\subsection{Situational Awareness - SART}

The results of the SART showed a very significant difference overall, and for several dimensions of the questionnaire. As all samples showed non-normal distribution, we performed only non-parametric tests. For the overall score, Scene interruptions produced very significantly higher values than the baseline $(p<$ $0.001)$ and Blank interruptions $(p=0.0002)$, while the difference between baseline and Blank was not significant $(p=0.14)$. Similarly, looking at the separate dimensions of the SART, subjects rated the complexity of the situation close to significantly lower with Scene interruptions, as compared to both, the baseline ( $W=329, p=.05207)$, and Blank ( $W=166, p=$ 0.08074). Arousal was also significantly higher for Scene interruptions compared to the baseline ( $W=289.5, p=0.02075$ ), and close to significantly higher compared to the Blank condition $(W=471.5, p=0.06857)$. Subjects rated the concentration of attention significantly higher in the Scene condition than for the baseline $(W=173.5, p=0.0005733)$, and for Blank interruptions ( $W=486.5, p=0.01986$ ). The division of attention, similarly, showed significantly higher responses for the Scene condition compared to the baseline $(W=129.5, p=$ 
Improving Take-Over Quality in Automated Driving By Interrupting Non-Driving Tasks

0.001099) and the Blank condition $(W=129.5, p=0.001099)$. Participants rated the information quality significantly higher for Scene interruptions than for the baseline $(W=129, p=$ 3.901e-07), and also compared to Blank interruptions ( $W=922, p$ $=6.946 \mathrm{e}-05)$.

\subsection{Task Performance - Error Rate}

4.3.1 Movie Task. There was no significant difference in the ratio of wrong answers between conditions, but within the two treatments in relation to the interruption. All wrong answer ratios were non-normally distributed.

A Friedman test showed no significant difference between conditions $\left(\chi^{2}=.38235, p=.826\right)$. Pairwise one-tailed comparisons between baseline $(M=.15, S D=.15)$ and Scene $(M=.13, S D=.13)$ did not show a significant difference $(W=$ $82.5, p=.2286$ ), neither did the difference between baseline and Blank $(M=.16, S D=.17 ; W=53, p=.3472)$, or between the two treatments $(W=86, p=.1764)$.

For different points of interruptions, there was a significant difference in the wrong answer ratio. The ratio was significantly higher for questions concerning events right after an interruption $(M=0.21, S D=.29)$ as compared to events between $(M=0.09, S D=.19 ; W=78, p=.004874)$, or before interruptions $(M=.15, S D=.2 ; W=165, p=.03123)$. There was also a significant difference for events before and between interruptions ( $W=196, p=.03744)$.

4.3.2 LMT Task. For the LMT task, there was a significant effect between conditions in the pairwise one-tailed comparison. The baseline produced a significantly higher wrong answer ratio $(M=0.03, S D=.03)$ than the Scene condition $(M=0.02, S D=.03$; $W=229, p=.03788)$, and close to significantly higher ratio compared to the Blank condition $(M=.02, S D=.02 ; W=190, p=$ .05863). There was no significant difference between the two treatments $(W=130, p=.4098)$.

\subsection{Task Load - NASA-TLX}

As most samples were non-normally distributed for Movie, we only performed non-parametric tests, with pairwise one-tailed comparisons.

4.4.1 Movie Task. For the Movie task (see the top of Table 1), there was a significant difference between conditions in terms of load, according to the NASA-TLX (Friedman $\chi^{2}=6.4272, p=$

\begin{tabular}{|c|c|c|c|c|c|c|c|c|}
\hline & & Mental D. & Physical D. & Temporal D & Perform. & Effort & Frustration & Overall \\
\hline \multirow{3}{*}{$\frac{0}{3}$} & Baseline & $\mathbf{3 3 . 0 8} \mid 22.94$ & $\mathbf{8 . 6 5} \mid 8.31$ & $\mathbf{1 9 . 4 2} \mid 15.51$ & $25.38 \mid 21.40$ & $\mathbf{3 1 . 1 5} \mid 22.55$ & $29.23 \mid 29.55$ & 24.49|14.67 \\
\hline & Scene & $39.23 \mid 23.44$ & $\mathbf{1 1 . 5 4} \mid 10.37$ & $28.85 \mid 20.94$ & $29.42 \mid 23.34$ & $\mathbf{3 5 . 1 9} \mid 25.00$ & $\mathbf{3 1 . 1 5} \mid 24.59$ & $29.23 \mid 14.99$ \\
\hline & Blank & 42.31 $\mid 23.50$ & $\mathbf{1 2 . 3 1} \mid 14.02$ & $\mathbf{2 8 . 4 6} \mid 24.61$ & $27.69 \mid 24.46$ & $\mathbf{3 8 . 0 8} \mid 22.94$ & $\mathbf{3 1 . 5 4} \mid 30.65$ & $\mathbf{3 0 . 0 6} \mid 16.52$ \\
\hline \multirow{3}{*}{$\sum_{\leftrightarrows}^{\ominus}$} & Baseline & $\mathbf{5 2 . 7 8} \mid 27.12$ & $23.33 \mid 19.36$ & $\mathbf{4 5 . 3 7} \mid 24.65$ & $27.04 \mid 18.67$ & $\mathbf{5 2 . 9 6} \mid 26.50$ & $\mathbf{4 2 . 7 8} \mid 26.18$ & 40.71 $\mid 16.00$ \\
\hline & Scene & $\mathbf{5 3 . 8 9} \mid 25.09$ & $20.19 \mid 18.05$ & $\mathbf{4 0 . 0 0} \mid 22.62$ & $25.74 \mid 18.64$ & $\mathbf{5 2 . 4 1} \mid 22.80$ & $\mathbf{3 3 . 5 2} \mid 21.48$ & $37.62 \mid 15.25$ \\
\hline & Blank & $\mathbf{4 7 . 4 1} \mid 22.33$ & $\mathbf{1 9 . 6 3} \mid 17.32$ & $\mathbf{4 2 . 4 1} \mid 22.89$ & $\mathbf{2 8 . 1 5} \mid 20.39$ & $\mathbf{4 7 . 2 2} \mid 23.47$ & $\mathbf{3 4 . 8 1} \mid 21.33$ & $\mathbf{3 6 . 6 0} \mid 13.91$ \\
\hline
\end{tabular}

Table 1. Descriptive results of the NASA-TLX dimensions for each condition, separated by task (Movie, LMT). The left value in bold font weight indicates the mean, the right value in regular font weight indicates the standard deviation.
.04021). The baseline load is significantly smaller than the overall load in Scene (Wilcoxon $W=84.5, p=.0185)$ and in Blank $(W=$ 103.5, $p=.03468)$; there was no significant difference between Scene and Blank $(W=160.5, p=.3563)$. For the single dimensions of the NASA-TLX, the baseline results were consistently smaller than either of the treatments. However, the difference was only significant (or close to it) for the demand dimensions, with no significant difference between the two treatments ever. Mental demand during the baseline was close to significantly smaller than with Scene interruptions ( $W=80.5, p=.06908)$, and significantly smaller than with Blank interruptions $(W=103.5$, $p=.03468$; Blank vs. Scene $W=160.5, p=.3563$ ). Physical demand during the baseline was close to significantly smaller than with Scene ( $W=15.5, p=.06323)$, and significantly smaller than with Blank interruptions ( $W=6, p=.01483$; Blank vs. Scene significantly smaller than with Scene $(W=43.5, p=.01977)$, and with Blank interruptions ( $W=73, p=.0237$; Blank vs. Scene $W=89.5, p=.4391$ ). Pairwise comparisons between the three conditions did not show significant differences for performance, effort, and frustration.

4.4.2 LMT Task. For the LMT task (see the bottom of Table 1), the baseline drive produced a significantly higher load than Scene $(t(26)=1.868, p=.03654)$ and Blank interruptions; the separate dimensions of the NASA-TLX showed similar results, not all of them significant. Two third of the samples were normally distributed, one third was non-normally distributed. Consequently, we performed both, parametric and nonparametric tests; as tendencies always state the same, we report only parametric results. Pairwise comparisons showed significant differences in mental demand, effort, and frustration. While there was no significant difference for mental demand between baseline and Scene interruptions $(t(26)=-0.24734, p=$ .5967), the difference was close to significant between baseline and Blank interruptions $(t(26)=1.4413, p=.08072$; closer with non-parametric tests: $W=177, p=.0518)$, and significant between Blank and Scene interruptions $(t(26)=1.9211, p=$ $0.03287)$. For the effort dimension, there was again no significant difference between baseline and Scene $(t(26)=.1751, p=.4312)$, while there was a significant difference between baseline and Blank $(t(26)=2.2104, p=.01804)$, and between Blank and Scene $(t(26)=1.9578, p=.03053)$. In terms of frustration, there was a $W=20.5, p=.4282)$. Temporal demand during the baseline was 
significant difference between baseline and Scene $(t(26)=2.0349$, $\mathrm{p}=.02609)$, as well as between baseline and Blank $(t(26)=1.7666$, $p=0.04452)$, but not between Blank and Scene $(t(26)=-0.4156, \mathrm{p}$ $=.3406$ ).

When comparing the two different tasks, the LMT task showed consistently higher means than the Movie task, except for the performance and frustration dimensions. Differences for performance were not significant. For frustration, there was a significant difference between the baselines of Movie and LMT ( $W=476.5, p=0.01274$ ), but not significant difference for Scene $(W=388, p=.257)$ or Blank $(W=418, p=.1172)$.

\section{Discussion}

\subsection{Driving Performance}

Benefitting from the interruptions, our participants showed improved reaction times in every setting, confirming H1. This result, however, was only significant for the low-effort, passive Movie task with Scene interruptions. We hypothesize that, on the one hand, the Movie task was more prone to attentional degradation, while the LMT task generally kept people more alert, resulting in higher effects for the Movie task. And, on the other hand, the improved results from Blank to Scene interruptions indicate that the interruption alone might not be enough, but it is the re-attachment of the subject to the driving scene that matters.

\subsection{Situational Awareness}

Results for the situational awareness based on the self-reported SART dimensions indicated a strong contrast for Scene interruptions compared to the other two conditions, partly confirming H2. Most of these differences were of a positive nature, like a reduced complexity of the situation, heightened concentration, and, most importantly a higher quality of information. They also, however, indicated undesired side effects, like a higher division of attention.

\subsection{Task Performance}

For the Movie task, there was no significant difference between conditions, i.e., at a minimum, our interventions did not negatively impact task performance, contributing to our assumptions in H3. However, both treatments seem to have affected participants' receptivity immediately after the interruption, as questions about this part showed significantly more errors. We assume that subjects' focus on the task was removed momentarily due to the interruptions, indicating a resumption lag [24]. Especially the Scene condition may have created additional switching costs, as people kept processing what they saw. Our results also indicate a positive effect from the interruptions, as questions about events in between the $30 \mathrm{~s}-$ period between interruptions showed the most correct answers. Presumably, the interruptions rebooted their focus on the task, after the initial lag.
For the LMT task, the interruptions impacted performance even in a positive way. Since performing the LMT over several minutes can be straining, we assume that interruptions presented an opportunity for participants to rest for a moment and refocus. This result was further corroborated by the results of the NASA-TLX, which indicated positive effects in several dimensions and overall with interruptions.

\subsection{Task Load}

For both tasks, the interruptions had a significant effect on participants' subjective task load - results were opposed, however, depending on the task, providing a more reflected view on our assumptions in H3. For the low-effort Movie task, the interruptions created a significant increase in load, as they constituted a potentially undesired disruption of the otherwise passive and monotonous watching activity. From this perspective, it is understandable that participants reported a significantly higher mental and temporal demand with interruptions. However, for Movie subjects, there also seemed to be a negative bias towards the two treatments, as both also showed a significant increase in physical demand, and the treatments involved absolutely no manual intervention. In light of this, it is particularly noteworthy that still, there was no significant difference in the reported effort or frustration with interruptions.

In contrast to this, interruptions helped to reduce the load of the cognitively demanding LMT. In this setup, however, only Blank interruptions showed a significant reduction in mental demand and effort. We hypothesize that Scene interruptions, which, in theory, constitute a break from the LMT, still kept demands high, as participants had to process the complex driving scene instead. Nonetheless, both types of interruptions reduced frustration levels significantly. Interestingly, this is almost the only dimension, in which the NASA-TLX did not indicate much higher values for the LMT: while there still was a significant effect of the LMT on the baseline as compared to the Movie task, the interruptions seemed to balance out the level of frustration for participants.

\subsection{Limitations}

While the experimental setup proved complex, which opened up many avenues for smaller errors, one rather large limitation is our homogenous sample. Most participants were in their twenties, with a few subjects just before or after this; most of them had a university background, and we also assume a high affinity for technology. Ultimately, driving automation and strategies to remedy its adverse side effects need to cater to all demographics, independent of age, level of education and other limiting factors.

The practical applicability of the introduced interruptions is also in question. On first sight, they seem like a frequent annoyance, interrupting the non-driving task that the user wants to engage with. Results indicate, however, that for certain types of tasks, e.g., fast-paced, cognitively demanding tasks, they might constitute a welcome alternative and showed many 
positive effects in our study. In the end, the only other option might be frequent take-overs of control [21,35] - and in comparison, we would argue that our introduced interruptions are less disrupting and for many people the preferred alternative.

Lastly, regarding our measures and results, effects during real-world driving might differ. We would assume people to be more alert in the real world, resulting in, e.g., faster reaction times under all conditions. Since we primarily look at differences between conditions, not absolute numbers, we still consider our results to be robust. Similarly, self-reported measures like the NASA-TLX and SART may leave additional room for error and experimenter's bias. The NASA-TLX questionnaire is the defacto standard to measure cognitive load in many research areas and has been used for decades. For situational awareness, we considered more objective measures, like the SAGAT (Situation Awareness Global Assessment Technique) [10], but ultimately decided against it, as it would have meant active and frequent interventions into our study procedure, which might have counter-acted the out-of-loop problem and affected our results, while the SART is performed ex-post; its results were also very significant.

\section{Conclusion \& Future Work}

In this paper, we report on a study that aims to improve takeover quality in semi-automated driving by interrupting the nondriving task frequently with either a blank screen or a combination of different views from the driving scene. Results indicate an improved take-over in terms of reaction time (H1), when a low-effort, passive task was interrupted by the driving scene. This type of interruptions also improved subjects' reported situational awareness (H2), while not affecting task performance unfavorably (H3); they can have positive effects on the task as well. With this study, we demonstrate new ways to improve the take-over problem in practice. Especially, interrupting IVIS tasks with the driving scene from time to time may represent a less obtrusive way to keep drivers in the loop during semi-autonomous driving, than frequent take-overs [21,35].

Going forward, we still aim to gain more insights from the current study. As with many experiments, we collected a large amount of data and see more opportunities for analyses. First, we will consider additional measures to evaluate differences in the driving performance between conditions. Second, the LMT task alone produced a plethora of data points, which will help us investigate task performance in more detail. And third, we have more qualitative records from the study, from which we plan to formulate new hypotheses for future studies.

In this regard, we can already anticipate additional value in different forms of presenting the interruptions and different forms of their content. While our study did not suggest that the current form of intervention was completely rejected by participants, any widely successful solution benefits from a high user acceptance. Similarly, evaluating these treatments in different TOR scenarios, and especially investigating potential relationships between scenario and type of treatment seem valuable next steps in this line of research.

\section{ACKNOWLEDGMENTS}

We thank Anna Andreeva, Valerian Backeberg, Michael Gröschel, Jonas Freudenstein, and Moritz Hüther for their work on the project.

\section{REFERENCES}

[1] German Research Center for Artificial Intelligence (Dfki). OpenDS: Open Source Driving Simulation. Retrieved from https://opends.dfki.de/.

[2] William Acker. 1982. A computerized approach to psychological screeningThe Bexley-Maudsley Automated Psychological Screening and The BexleyMaudsley Category Sorting Test. International fournal of Man-Machine Studies 17, 3, 361-369. DOI: https://doi.org/10.1016/S0020-7373(82)80037-0

[3] Piotr D. Adamczyk and Brian P. Bailey. 2004. If not now when?: the effects of interruption at different moments within task execution. In Proceedings of the Proceedings of the SIGCHI Conference on Human Factors in Computing Systems. ACM Press, New York, NY, 271-278. DOI: https://doi.org/10.1145/985692.985727

[4] Charles E. Billings. 1991. Human-centered aircraft automation: A concept and guidelines. NASA Technical Report NASA-TM-103885, A-91192, NAS 1.15:103885. NASA Ames Research Center, Moffett Field, CA.

[5] D. Damböck, M. Farid, L. Tönert, and K. Bengler. 2012. Übernahmezeiten beim hochautomatisierten Fahren. In Proceedings of the Tagung Fahrerassistenz, 16.

[6] Joost C.F. De Winter, Riender Happee, Marieke H. Martens, and Neville A Stanton. 2014. Effects of adaptive cruise control and highly automated driving on workload and situation awareness: A review of the empirical evidence. Transportation Research Part F: Traffic Psychology and Behaviour 27, 196-217. DOI: https://doi.org/10.1016/j.trf.2014.06.016

[7] U.S. Department Of Defense. The DAPRA Grand Challenge. Retrieved from https://www.darpa.mil/about-us/timeline/-grand-challenge-for-autonomousvehicles.

[8] Birsen Donmez, Linda Ng Boyle, and John D Lee. 2006. The impact of distraction mitigation strategies on driving performance. Human Factors: The Journal of the Human Factors and Ergonomics Society 48, 4, 785-804. DOI: https://doi.org/10.1518/001872006779166415

[9] Amos Drory. 1985. Effects of rest and secondary task on simulated truckdriving task performance. Human Factors: The fournal of the Human Factors and Ergonomics Society 27, 2, 201-207. DOI: https://doi.org/10.1177/001872088502700207

[10] Mica R Endsley. 1988. Situation awareness global assessment technique (SAGAT). In Proceedings of the Aerospace and Electronics Conference. IEEE, 789795. DOI: https://doi.org/10.1109/NAECON.1988.195097

[11] Mica R. Endsley and Esin O. Kiris. 1995. The Out-of-the-Loop Performance Problem and Level of Control in Automation. Human Factors: The fournal of the Human Factors and Ergonomics Society 37, 381-394. DOI: $10.1518 / 001872095779064555$

[12] Geoffrey A. Fowler. 2018. Behind the wheel of a Tesla Model 3: It's a giant iPhone - for better and worse. Retrieved September 20, 2018 from https://www.washingtonpost.com/technology/2018/08/02/behind-wheel-teslamodel-its-giant-iphone-better-worse/.

[13] Pnina Gershon, Adi Ronen, Tal Oron-Gilad, and David Shinar. 2009. The effects of an interactive cognitive task (ICT) in suppressing fatigue symptoms in driving. Transportation Research Part F: Traffic Psychology and Behaviour 12, 1, 21-28. DOI: https://doi.org/10.1016/j.trf.2008.06.004

[14] Christian Gold, Daniel Damböck, Lutz Lorenz, and Klaus Bengler. 2013. "Take over!" How long does it take to get the driver back into the loop? Proceedings of the Human Factors and Ergonomics Society Annual Meeting 57, 1938-1942. DOI: $10.1177 / 1541931213571433$

[15] Paul Green. 2000. Crashes Induced by Driver Information Systems and What Can Be Done to Reduce Them. Society of Automotive Engineers Conference Proceedings 27-36.

[16] Sandra G. Hart and Lowell E. Staveland. 1988. Development of NASA-TLX (Task Load Index): Results of Empirical and Theoretical Research. Advances in Psychology 52, 139-183. DOI: 10.1016/S0166-4115(08)62386-9

[17] Sae International. 2016. 73016A: Taxonomy and Definitions for Terms Related to Driving Automation Systems for On-Road Motor Vehicles. Society of Automotive Engineers, Troy, MI.

[18] S. Langlois and B. Soualmi. 2016. Augmented reality versus classical HUD to take over from automated driving: An aid to smooth reactions and to anticipate maneuvers. In Proceedings of the IEEE Conference on Intelligent Transportation Systems. IEEE, 1571-1578. DOI: 10.1109/ITSC.2016.7795767 
[19] Yi-Ching Lee, John D. Lee, and Linda Ng Boyle. 2007. Visual Attention in Driving: The Effects of Cognitive Load and Visual Disruption. Human Factors: The fournal of the Human Factors and Ergonomics Society 49, 4, 721-733. DOI: $10.1518 / 001872007 X 215791$

[20] Tyron L. Louw. 2017. The Human Factors of Transitions in Highly Automated Driving. Ph.D. Dissertation. University of Leeds, Leeds, UK.

[21] Tyron L. Louw, Natasha Merat, and Hamish Jamson. 2015. Engaging with highly automated driving. To be or not to be in the loop. In Proceedings of the International Driving Symposium on Human Factors in Driver Assessment, Training and Vehicle Design, 190-196. DOI: 10.13140/RG.2.1.2788.9760

[22] Jaimie C. Mcnabb. 2017. Warning a Distracted Driver: Smart Phone Applications, Informative Warnings and Automated Driving Take-Over Requests. Ph.D. Dissertation. Arizona State University, Tempe, AZ.

[23] Natasha Merat and A. Hamish. Jamson. 2009. How do drivers behave in a highly automated car? In Proceedings of the International Driving Symposium on Human Factors in Driver Assessment, Training and Vehicle Design, 514-521. DOI: 10.17077/drivingassessment.1365

[24] Christopher A. Monk, Deborah A. Boehm-Davis, George Mason, and J. Gregory Trafton. 2004. Recovering From Interruptions: Implications for Driver Distraction Research. Human Factors: The Journal of the Human Factors and Ergonomics Society 46, 650-663. DOI: 10.1518/hfes.46.4.650.56816

[25] Christopher A. Monk, Deborah A. Boehm-Davis, and J. Gregory Trafton. 2002. The Attentional Costs of Interrupting Task Performance. Proceedings of the Human Factors and Ergonomics Society Annual Meeting 46, 22, 1824-1828. DOI: https://doi.org/10.1177/154193120204602210

[26] Christopher A. Monk, J. Gregory Trafton, and Deborah A. Boehm-Davis. 2008 The Effect of Interruption Duration and Demand on Resuming Suspended Goals. Fournal of Experimental Psychology: Applied 14, 4, 299-313. DOI: $10.1037 / \mathrm{a} 0014402$

[27] Frederik Naujoks. 2017. The Importance of Interruption Management for Usefulness and Acceptance of Automated Driving. In Proceedings of the Automotive User Interfaces and Interactive Vehicular Applications. ACM DOI: $10.1145 / 3122986.3123000$

[28] Catherine Neubauer, Gerald Matthews, Lisa Langheim, and Dyani Saxby. 2012. Fatigue and voluntary utilization of automation in simulated driving. Fatigue and voluntary utilization of automation in simulated driving 54, 5, 734746. DOI: $10.1177 / 0018720811423261$

[29] Robert J. Nowosielski. 2017. Using Audiobooks to combat Mental Underload: How Traffic Density and Road Complexity affect Driving Performance while Multitasking in Virtual Environments. M.Sc. Thesis. University of Guelph, Ontario, Canada.

[30] Sebastiaan Petermeijer, Pavlo Bazilinskyy, Klaus Bengler, and Joost De Winter. 2017. Take-over again: Investigating multimodal and directional TORs to get the driver back into the loop. Applied Ergonomics 62, 204-215. DOI: 10.1016/j.apergo.2017.02.023

[31] Ioannis Politis, Stephen Brewster, and Frank Pollick. 2015. Language-based multimodal displays for the handover of control in autonomous cars. In Proceedings of the International Conference on Automotive User Interfaces and Interactive Vehicular Applications, 3-10. DOI: 10.1145/2799250.2799262
[32] J. Radlmayr, C. Gold, L. Lorenz, M. Farid, and K. Bengler. 2014. How Traffic Situations and Non-Driving Related Tasks Affect the Take-Over Quality in Highly Automated Driving. Proceedings of the Human Factors and Ergonomics Society Annual Meeting 58, 2063-2067. DOI: 10.1177/1541931214581434

[33] Neville A. Stanton and Philip Marsden. 1996. From Fly-by-Wire to Drive-byWire: Safety implications of automation in vehicles. Safety Science 24, 1, 35-49. DOI: https://doi.org/10.1016/S0925-7535(96)00067-7

[34] R. M. Taylor. 1990. Situational awareness rating technique (SART): The development of a tool for aircrew systems design. Situational awareness in aerospace operations 1-17.

[35] Silvia F Varotto, Raymond G Hoogendoorn, Bart Van Arem, and Serge P. Hoogendoorn. 2014. Human Factors of Automated Driving: Predicting the Effects of Authority Transitions On Traffic Flow Efficiency. In Proceedings of the TRAIL Internal PhD Conference, 1-16.

[36] Guido Wager, Mark P Mchenry, Jonathan Whale, and Thomas Bräunl. 2014 Testing Energy Efficiency and Driving Range of Electric Vehicles in Relation to Gear Selection. Renewable Energy 62, 303-312. DOI: 10.1016/j.renene.2013.07.029

[37] Marcel Walch, Kristin Lange, Martin Baumann, and Michael Weber. 2015 Autonomous driving: Investigating the Feasibility of Car-Driver Handover Assistance. In Proceedings of the International Conference on Automotive User Interfaces and Interactive Vehicular Applications, 11-18. DOI: $10.1145 / 2799250.2799268$

[38] Nicole E. Werner, Cyrus K. Foroughi, Carryl Baldwin, Robert Youmans, and Deborah A. Boehm-Davis. 2018. Associative activation during interrupted task performance: a mixed methods approach to understanding the overall quality effects of interruptions. Theoretical Issues in Ergonomics Science 19, 118-134. DOI: $10.1080 / 1463922 X .2017 .1284282$

[39] Earl L. Wiener and Renwick E. Curry. 1980. Flight-deck automation: Promises and problems. Ergonomics 23, 995-1011.

[40] Felix Wulf, Kathrin Zeeb, Maria Rimini-Doring, Marc Arnon, and Frank Gauterin. 2013. Effects of human-machine interaction mechanisms on situation awareness in partly automated driving. In Proceedings of the IEEE Conference on Intelligent Transportation Systems, 2012-2019. DOI: 10.1109/ITSC.2013.6728525

[41] Kathrin Zeeb. 2017. Der Einfluss fahrfremder Tätigkeiten auf die Fahrerübernahme während des hochautomatisierten Fahrens. Ph.D. Dissertation. Heinrich-Heine-Universität Düsseldorf,

[42] B. Zeigarnik. 1938. On finished and unfinished tasks. A source book of Gestalt psychology 1, 300-314.

[43] Fred R H Zijlstra, Robert A Roe, Anna B Leonora, and Irene Krediet. 1999. Temporal factors in mental work: Effects of interrupted activities. Fournal of Occupational and Organizational Psychology 72, 2, 163-185. DOI: $10.1348 / 096317999166581$ 